\title{
Osteolisis multicéntrica
}

\section{Anta Martínez, S. Pombo Expósito, A. Vieitez Rey, J. J. Suárez Gestal, A. González-Carreró Sixto, J. González García ${ }^{(1)}$}

Servicio de Cirugía Ortopédica y Traumatología. Complejo Hospitalario Universitario de Santiago de Compostela. A Coruña. EsPaña

(1) MÉdicos ADJUNTOS

Personal Docente Colaborador del Departamento de Cirugía. Universidad de Santiago de Compostela. A CORUÑa. ESPAÑa.

\section{Correspondencia:}

Dra. Laura Anta Martínez

Hospital Universitario de Santiago de Compostela

Servicio COT

Avda. Choupana, s/n

15706 Santiago de Compostela

A Coruña (España)

Teléfono: 0034661914612

e-mail: laurianta@hotmail.com

Se presenta un caso de osteolisis carpo-tarsal multicéntrica, tipo de displasia esquelética extraordinariamente infrecuente, en una paciente de veintiún años de edad. El correcto diagnóstico de la enfermedad puede evitar tratamientos innecesarios.

Palabras clave: osteolisis multicéntrica, displasia esquelética, enfermedad ósea
We present a case of multicentric carpo-tarsal osteolysis, a extremely rare skeletal dysplasia in a patient of twenty-years of age. A correct diagnostic of the disease may avoid unnecessary treatments.

Key words: multicentric ostheolysis, skeletal dysplasia, bone disease

\section{INTRODUCCIÓN}

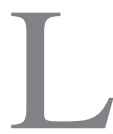

a osteolisis carpo-tarsal multicéntrica (OCTM), es una enfermedad extraordinariamente infrecuente, pues desde que fue descrita en 1832 por Jackson ${ }^{1}$, no han sido publicados en la literatura más de cien casos ${ }^{2,3}$. Esta peculiar displasia esquelética puede ir, o no, asociada con un síndrome nefrótico, el cual puede condicionar la esperanza de vida del paciente. Con respecto al tratamiento, debe ir encaminado a prevenir inestabilidades articulares y minimizar el dolor con la administración de analgésicos/antiinflamatorios, fármacos que no alteran la progresión de la enfermedad. En el presente trabajo, se da a conocer un nuevo caso de OCTM y se hace una revisión de esta particular displasia.

\section{CASO CLÍNICO}

Paciente de 21 años de edad, diagnosticada en la infancia de osteolisis carpo-tarsiana idiopática, sin signos de afectación renal. Los padres y un hermano no presentaban patología alguna.

A los cuatro años había sido intervenida de ambos pies cavos en otro centro, siendo reintervenida en nuestro centro a los 13 años por recidiva de la deformidad en el pie derecho. A los 14 años se le practicó una artroscopia de rodilla, observándose gran destrucción articular con irregularidades óseas a nivel de metáfisis distal de fémur.

La paciente presentaba deformidad adaptativa del carpo bilateral con desviación cubital y limitación para la movilidad de muñeca y dedos, con desaparición total de huesos del carpo 


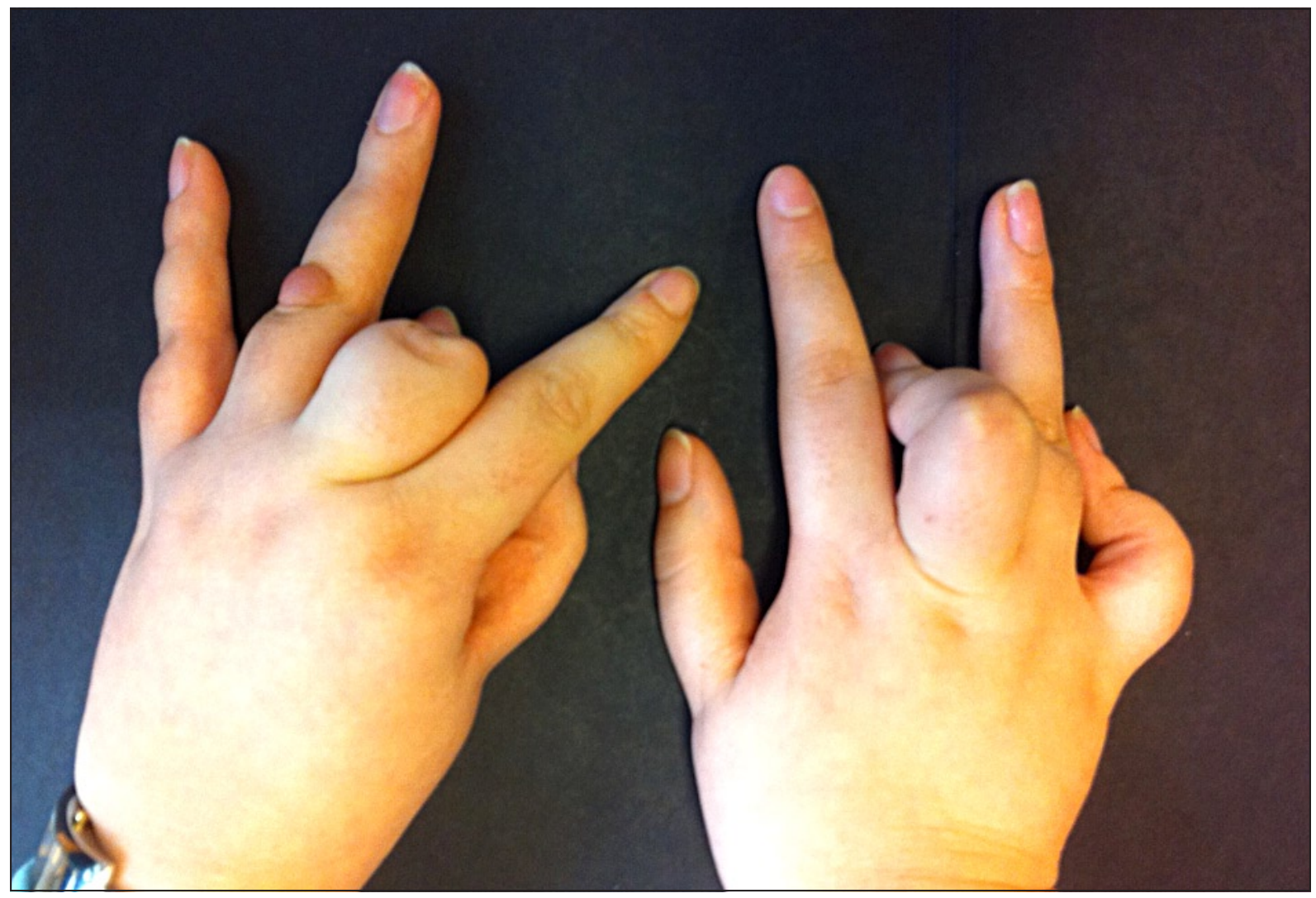

Figura 1. Fotografía de las manos de la paciente.

y adelgazamiento de huesos tubulares, metacarpianos y metáfisis distal de cúbito, con adaptación del radio distal. Asimismo, presentaba en el tercer dedo mano izquierda y en el tercer y quinto dedos mano derecha, una deformidad fija en ojal (boutonnière), secundaria a anquilosis interfalángica proximal y luxación interfalángica distal. Las articulaciones metacarpofalángicas estaban subluxadas bilateralmente, encontrándose en el tercer dedo de la mano izquierda, una tumoración de origen fibroso (Figuras 1 y 2).

El pie izquierdo presentaba deformidad en cavo con inclinación calcánea de $45^{\circ}$, y artrodesis de las articulaciones subastragalina, astragalo-escafoidea, escafo-cuneana, cuneo-metatarsiana-primer radio y calcaneo-cuboidea. El $1^{\circ}$ y $4^{\circ}$ dedos presentaban deformidad en martillo irreductible, y el $5^{\circ}$ dedo artrodesis de la interfalángica distal.

El pie derecho se había artrodesado a nivel de articulaciones subastragalina, astrágalo-escafoidea, escafo-cuneana, cuneo-metatarsiana-primer radio y calcaneo-cuboidea. El $1^{\circ}$ y $5^{\circ}$ dedos en martillo eran rígidos, con artrodesis metatarsofalángica-primer dedo y subluxación metatarsofalángica del resto de dedos. Existía osteolisis de la base de metatarsianos y cabezas de las falanges proximales del $2^{\circ}-5^{\circ}$ dedos. (Figura 3 )

En los datos del laboratorio, los reactantes de fase aguda sugestivos de proceso inflamatorio permanecían normales, así como los parámetros de la función renal.

\section{DISCUSIÓN}

En 1838 Jackson describió por primera vez la enfermedad con el nombre de «brazo sin hueso» (boneless arm), aunque han sido utilizados otros términos en la literatura para describirla, como osteolisis esencial, osteolísis esencial progresiva, acro-osteolisis idiopática, osteolisis hereditaria, osteolisis hereditaria multicéntrica, agenesia carpotarsal, disostosis carpal familiar y necro- 

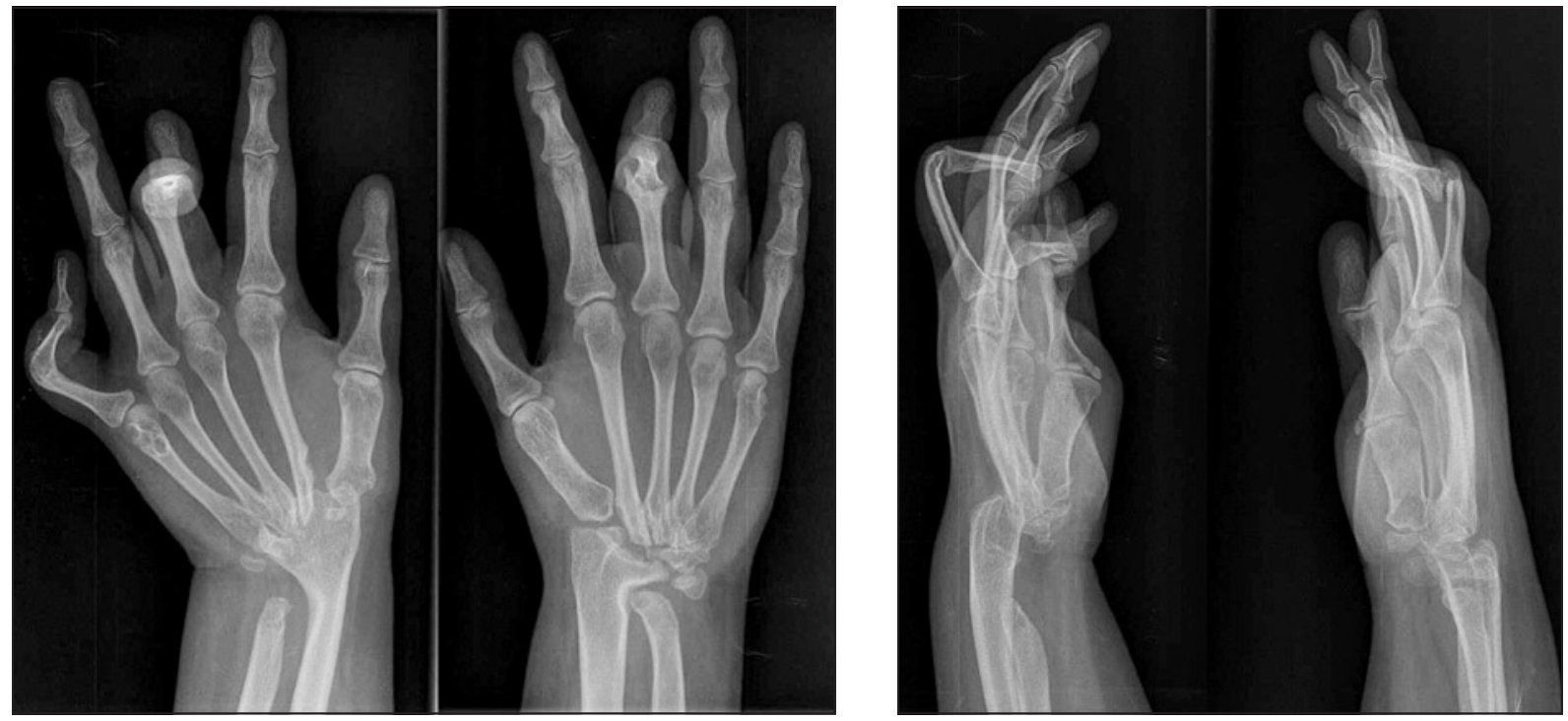

Figura 2. RX. Desaparición total de huesos del carpo y adelgazamiento de metacarpianos y metáfisis distal de cúbito lo que provoca una marcada desviación cubital con adaptación de radio distal.

sis bilateral carpal ${ }^{1,4,5}$. Sin embargo, desde que Thieffry y Sorrel-Dejerine en $1959^{6}$, presentaron tres generaciones de una misma familia con antecedentes de episodios de artritis aguda en manos, reabsorción de los huesos del carpo y tarso, así como una progresión al desarrollo de pies cavos, se la conoce como osteolisis multicéntrica carpo-tarsal. La asociación de la enfermedad con una insuficiencia renal grave fue descrita por primera vez por Marie et al. en $1951^{7}$.
Torg et al. en 1969, clasificaron la osteolisis en 4 tipos $^{8}$ : 1) osteolisis masiva de Gorham; 2) osteolisis esencial multifocal con nefropatía; 3) osteolisis idiopática hereditaria con trasmisión autosómica dominante; y 4) osteolisis idiopática hereditaria con trasmisión autosómica recesiva. MacPherson et al. añadieron un quinto tipo: el síndrome de Winchester ${ }^{9,}{ }^{10}$. Sin embargo, Tyler y Rosenbaum ${ }^{11}$ agruparon estos términos en uno solo, la osteolisis multicéntrica idiopática,
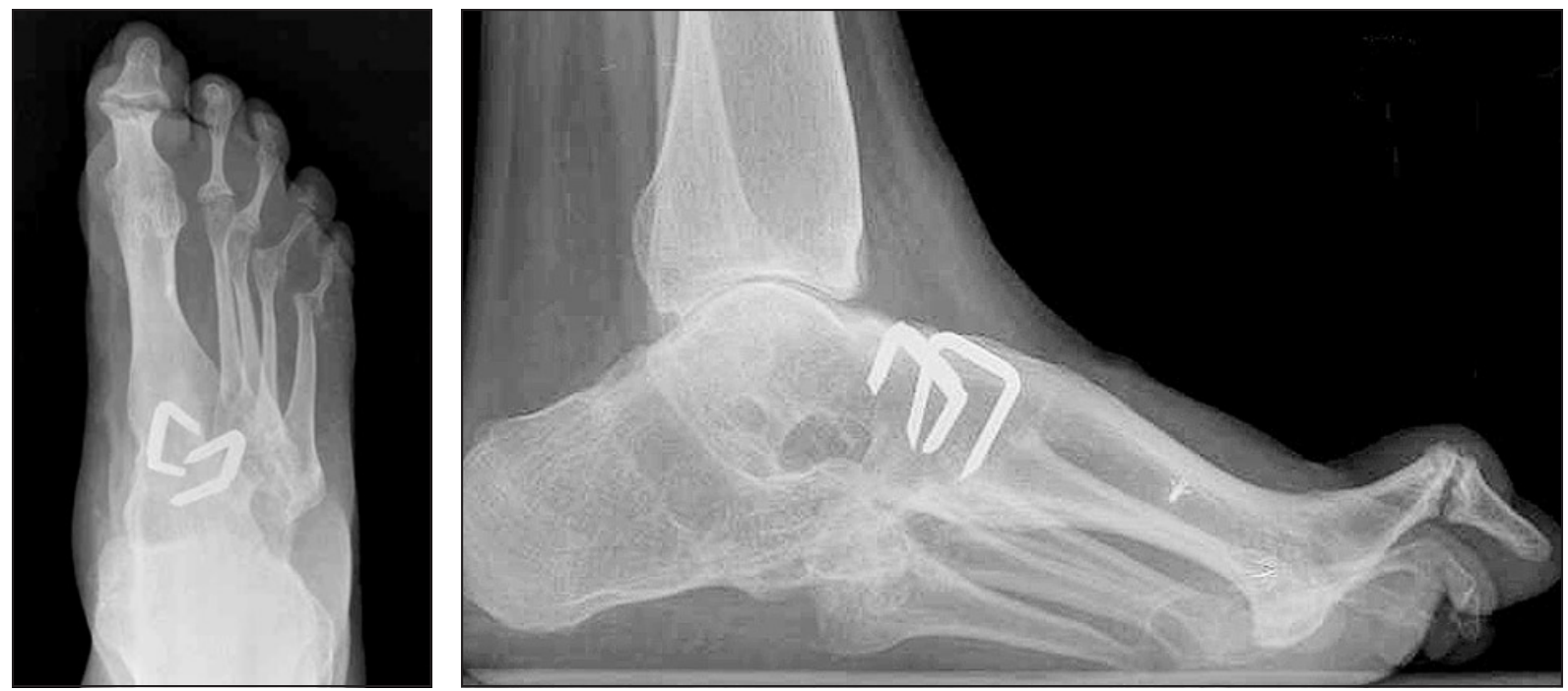

Figura 3. Artrodesis del retro- y medio pies por pies cavos. Osteolisis de la base de metatarsianos y cabezas de las falanges proximales del $2^{\circ}-5^{\circ}$ dedos. 
que dividieron en 2 grupos principales: osteolisis multicéntrica con nefropatía y osteolisis multicéntrica hereditaria. Aquellos con nefropatía tenían proteinuria e insuficiencia renal, con resultado de muerte en la tercera década. No tenían antecedentes familiares ni de osteolisis carpo-tarsal ni de fallo renal. El segundo grupo lo definía claramente su presentación familiar, con patrón de herencia autosómico dominante o recesivo. La osteolisis masiva de Gorham no se incluía por su carácter unicéntrico, mientras que el síndrome de Winchester, también se excluyó por su presentación clínica diferente, y porque la osteolisis carpo-tarsal se acompañaba de una importante osteoporosis generalizada y destrucción total de otras articulaciones, simulando la artritis reumatoide. Recientemente, la International Bone Dysplasia Society ha clasificado estas enfermedades en cuatro grupos, en base a sus características clínicas y radiológicas, así como a su modo de herencia (Tabla I) ${ }^{5}$.

La osteolisis multicéntrica carpo-tarsal con nefropatía, se caracteriza por un debut temprano a los 2-3 años de vida, con clínica de dolor e inflamación en muñecas y/o pies, con progresiva desaparición de los huesos del carpo y tarso, con afectación más marcada a nivel del carpo ${ }^{2,12}$. Los huesos tubulares adyacentes también se ven afectados. Así, los metacarpianos se presentan adelgazados en su extremo proximal, con desaparición de su base, y el cúbito suele acortarse proximal y distalmente, con longitud del radio normal, lo que provoca una característica desviación cubital de la muñeca ${ }^{3}$. La enfermedad, de la que no se ha podido demostrar su mecanismo patogenético exacto, se estabiliza en la adolescencia. Los casos que se acompañan de nefropatía grave suelen morir entre la tercera o cuarta décadas de la vida ${ }^{4,5,11,13-17}$. También es frecuente la asociación con malformaciones congénitas del pie, como pies cavos o zambos, como en el caso que presentamos. La característica diferencial con la osteolisis multicéntrica hereditaria, además de su patrón de herencia, es quizá la afectación de los huesos tubulares adyacentes ${ }^{4}, 10,14,15,18$.

El caso que se presenta se clasificaría como osteolisis carpo-tarsal multicentrica (OCTM) con/sin nefropatía. En este último aspecto, es importante destacar que la ausencia de nefropatía en el momento actual no excluye su desarrollo posterior, pues se han descrito en la literatura casos en los que la nefropatía puede estar latente durante un largo periodo de tiempo ${ }^{7,18,19}$.

La patogenia de la enfermedad, en todos sus tipos, es desconocida 2, 4, 14, 16, 17, 20. La clínica y

Tabla I - Clasificación de la International Bone Dysplasia Society 5

\begin{tabular}{|l|c|c|c|}
\hline $\begin{array}{l}\text { Osteocondrodisplasias } \\
\text { Grupo 31: Osteolisis }\end{array}$ & Herencia & $\begin{array}{c}\text { Presente } \\
\text { nacimiento }\end{array}$ & Cromosoma \\
\hline Multicéntrica, predominantemente carpal y tarsal & & & \\
Osteolisis multicéntrica carpo-tarsal con y sin nefropatía (MCTO) & AD & - & \\
Osteolisis carpo-tarsal de Shinohara & AR & - & \\
\hline Multicéntrica predominantemente carpal, tarsal e interfalángica & AR & - & \\
Síndrome de Francois & AR & - & \\
Síndrome de Winchester & AD & - & \\
Síndrome de Torg & & & \\
Osteolisis carpo-tarsal falángica de Whyte Hemingway & AD & - & \\
\hline Predominantemente de falanges distales & AR & - & \\
Síndrome de Hadju-Cheney & AR & - & \\
Acroosteolisis neurogénica familiar de Giacci & & & \\
Síndrome mandibulo acral & AD & - & $1 \mathrm{Q} 21.2$ \\
\hline Afectación predominante de diáfisis y metáfisis & AR & - & $18 Q 21.1-q 22$ \\
\hline Osteolisis expansiva familiar & \\
Fibromatosis hialina juvenil &
\end{tabular}


la progresión indican un mecanismo patogénico común. Las biopsias renales efectuadas en diversos casos, han mostrado glomeruloesclerosis focal segmentaria con atrofia tubular, fibrosis intersticial e infiltración celular progresando a hialinización de la mayoría de los glomerulos ${ }^{13}$. Lagier y Rutishauer concluyeron que la patología vascular no era la primera causa, ni de la nefropatía ni de la osteolisis ${ }^{20}$. Muyshondt presentó un caso de un paciente afecto de osteolisis tipo III en el que existió una reactivación de la osteolisis a nivel de codo izquierdo, con dolor e incapacidad, tras realizar una fistula arteriovenosa braquial izquierda como tratamiento de su afectación renal. No pudo demostrar su hipótesis de que la isquemia inducida por la fístula arterio-venosa braquial izquierda (fenómeno de robo) podría estar implicada en el desencadenamiento de la osteolisis de los huesos del $\operatorname{codo}^{21}$.
Finalmente Tyler y Rosenbaum sugirieron una etiología mediada por complejos autoinmunes ${ }^{11}$, aunque no se ha podido confirmar.

La progresión de la enfermedad no se ve alterada por ninguna terapia, antiinflamatorios o corticoides. El tratamiento debe ser de soporte, para prevenir inestabilidades articulares y analgésicos/antiinflamatorios con el único fin de aliviar el dolor. La rehabilitación puede ser beneficiosa. La cirugía no frena el curso natural de la enfermedad, aunque ésta se estabiliza en la segunda-tercera décadas de la vida ${ }^{3,10,15}$.

El principal diagnóstico diferencial debe realizarse con la artritis crónica juvenil, por su debut en la infancia, y con dolor e inflamación de pequeñas articulaciones, aunque no existe afectación sistémica y los reactantes de fase aguda se encuentran dentro de los límites normales ${ }^{22-26}$.

\section{BIBLIOGRAFÍA}

1. Jackson JBS. A boneless arm. Boston Med Surg J, 1838; 18: 368-9.

2. Urlus $\mathrm{M}$, De Smet L, Fabry G, Lammens J, Molenaers G, Roosen $\mathrm{P}$, et al. Carpo-tarsal osteolysis case report and review of the literature. Genet Counsel, 1993; 4: 25-36.

3. Pai Gs, MacPherson RI. Idiopathic multicentric osteolysis: report of two new cases and a review of the literature. Am J Med Genet, 1988; 29: 929-36.

4. Hirooka M, Hirota M. Chronic nephropathy in idiopathic multicentric osteolysis. Int J Pediatr Nephrol, 1985; 6: 145-50.

5. Lachman RS, International nomenclature and classification of the osteochondrodysplasias. Pediatr Radiol, 1998; 28: 73744.

6. Thieffry S, Sorrell-Dejerine J. Forme speciale d'osteolyse essentielle héréditaire et familiale à stabilisation spontanée survenant dans l'enfance. Presse Méd, 1958; 66: 1858-61.

7. Marie J, Salet J, Leveque B. Polystrophies squelettiques avec osteolyse progressive. Arch Franç Pédiatr, 1951; 8: 752-3.

8. Torg JS, DiGeorge AM, Kirkpatrick JA, Trujillo M. Hereditary multicentric osteolysis with recessive transmission: a new syndrome. J Pediatr, 1969; 75: 243-52.

9. Eisenstein DM, Poznanski AK, Pachman LM. Torg osteolysis syndrome. Am J Med Genet, 1980; 80: 207-12.

10. Ros P, Pelaez E, Gallego N, Corbatn J, Ortuno J. Carpal osteolysis: An unusual enity treated with renal transplantation. Nephron, 1990; 55: 434-5.

11. Tyler T, Rosenbaum HD: Idiopathic multicentric osteolysis. AJR, 1976; 126: 23-31.

12. Hardegger F, Segmueller G, Simpson LA. The syndrome of idiopathic osteolysis. J Bone Joint Surg B, 1985; 67: 89-93.

13. Bakker SJL, Mulder AH, Tiebosch TMG, Verschure PDMM, Vos GD. Abnormal glomerular basement membrane in idiopatic multicentric osteolysis. Pediatr Nephrol, 1996; 10: 200-2.

14. Bennett WM, Beals $C$, Houghton
DC. Nephropathy of idiopathic multicentric osteolysis. Nephron, 1980; 25: 134-8.

15. Borra SI, Keinfeld M. A patient with carpotarsal osteolysis and nephropathy treated with longterm hemodialysis. Am J Kidney Dis, 1995; 26: 788-91.

16. Chiang HW, Chu TS, Tsai CC, Hsieh BS. Idiopathic multicentric osteolysis with nephropathy. J Formos Med Assoc, 2000; 99: 243-7.

17. Dickson GR, Carr KE, Davis R, Hamilton A, Hayes D, Mollan RAB. An investigation of vanishing bone disease. Bone, 1990; 11: 205-10.

18. Derot M, Rahtery M, Rosselin G, Catellier C. Acrostéolyse du carpe, pied creux, scoliose et strabismus chez une jeune fille atteinte d'une insuffisance renale. Bull Mém Soc Méd Hôp Paris, 1961; 77: 223-8.

19. Berthoux F, Robert JM, Zech P, Fries D, Traeger J. Acroosteolyse essentielle. À debut carpien et tarsien avec nephropathie. Arch Fr Pediatr, 1971; 28: 615-30. 
20. Lagier RL, Rutishauer E. Osteoarticular changes in a case of essential osteolysis. An anatomical and radiological study. J Bone Joint Surg B, 1965; 47: 339-53.

21. Muyshondt I, Lateur L, Van Roost G, Maes B. Osteolysis induced by AV-fistula in idiopathic carpotarsal osteolysis. Nephrol Dial Transplant, 2003; 18: 2185-8.

22. Carnevale A, Canun S, Del Castillo V, Mendoza L. Idiopa- thic multicentric osteolysis with facial anomalies and nephropathy. Am J Med Genet, 1987; 26: 877-86.

23. Faber MR, Verlaak R, Fiselier TJW, Hamel BCJ, Franssen MJAM, Gerrits GPJM. Inherited multicentric osteolysis with carpal-tarsal localisation mimicking juvenile idiopathic arthritis. Eur J Pediatr, 2004; 163: 612-8.

24. Petty RE, Southwood TR, Baum J, Bhettay E, Glass DN,
Manners P, et al. Revision of the proposed classification criteria for juvenile idiopathic arthritis: Durban 1997. J Rheumatol, 1998; 25: 1991-4.

25. Tuncbilek E, Bakkaloglu A, Besim A, Secmeer G, Tuncer E. Carpal-tarsal osteolysis. Pediatr Radiol, 1985; 15: 255-8.

26. Whyte MP, Avioli LV, Kleerekoper M, Murphy WA, Teitelbaum SL. Idiopathic multicentric osteolysis. Arthritis Rheum, 1978; 21: 367-76. 